\title{
An Observational Study Evaluating the Performance of LENT Score in the Selected Population of Malignant Pleural Effusion from Lung Adenocarcinoma in Singapore
}

\author{
John Abisheganaden ${ }^{a} \quad$ Akash Verma $^{a}$ Rucha S. Dagaonkar ${ }^{a}$ Richard W. Light ${ }^{b}$ \\ a Department of Respiratory and Critical Care Medicine, Tan Tock Seng Hospital, Singapore, Singapore; \\ b Pulmonary Disease Program, Vanderbilt University Hospital, Nashville, TN, USA
}

\section{For editorial comment see p. 303}

\section{Keywords}

Cancer (lung) · Pleural effusion · Survival · Epidermal growth factor receptor · Eastern Cooperative Oncology Group

\begin{abstract}
Background: Patients with malignant pleural effusion (MPE) secondary to lung cancer have been associated with poor prognosis historically. LENT score developed to riskstratify unselected patients with MPE predicts prognosis of $<6$ months in patients with lung cancer. Objective: To assess the performance of LENT score in predicting prognosis in selected population of MPE secondary to lung adenocarcinoma alone. Methods: A retrospective observational study was conducted by reviewing the medical records of patients managed for MPE in the year 2012. Results: Seventy patients with lung adenocarcinoma presenting with MPE were studied. The median (range) LENT score at initial diagnosis was 5 (2-7), and the median survival $7.9(0.13-40)$ months. Thirtynine patients received epidermal growth factor receptor-tyrosine kinase inhibitor (EGFR-TKIs). The median LENT score and median survival was 4 (2-7) and 14.4 months, respectively, in this group. Those in high-risk category by LENT in this group ( $n=19$ ) had a median survival and 6-month survival of 17.4 months and $73.6 \%$, respectively. Thirty-one pa-
\end{abstract}

\section{KARGER}

(c) 2018 S. Karger AG, Basel

E-Mail karger@karger.com

www.karger.com/res tients were treated with conventional chemotherapy. The median LENT score and median survival was 5 (2-7) and 4.1 (0.13-34.3) months, respectively, in this group. The median survival and 6-month survival rate in patients in highrisk category and moderate-risk category by LENT score was 6.2 months and $52.7 \%$, and 11.4 months and $70.5 \%$, respectively. Conclusion: LENT score underestimates prognosis in patients having MPE secondary to lung adenocarcinoma. This disparity particularly applies to the lung adenocarcinoma patients carrying EGFR mutation. Hence, LENT score may not be applicable to, or may need modification before applying to such patients.

(c) 2018 S. Karger AG, Basel

\section{Introduction}

Up to half of the patients with various metastatic malignancies develop para-malignant or malignant pleural effusion (MPE) $[1,2]$. In the case of lung malignancy, $15 \%$ have a pleural effusion at the initial diagnosis of lung cancer [3]. It is known that MPE recurs rapidly, sometimes within a month after an initial thoracentesis [2]. The management of these patients is thus twofold, one targeted at the cancer itself such as chemotherapy, radiotherapy or tyrosine ki- 
nase inhibitor (TKI), and the other targeted at the prevention of recurrence of MPE in future, after initial drainage, by employing chemical pleurodesis, tunneled pleural catheter, pleurectomy, or pleuroperitoneal shunt $[2,4-8]$.

Choice of these strategies hinges on predicted survival and presence or absence of lung entrapment. Ability to predict survival helps prioritize therapeutic options for MPE, enabling better match with patient's circumstances. Identifying patients with poorest survival carries potential for improved quality of life by shifting focus on minimizing discomfort and inconvenience toward the end of their life. Identifying patients with good survival on the other hand carries potential for improved quality of life by minimizing discomfort and morbidity associated with recurrence of MPE by providing prevention strategies like pleurodesis or tunneled pleural catheter. To this end, there are several factors that predict poor survival of unselected patients with MPE [9-13]. These are high pleural fluid lactate dehydrogenase (LDH; >1,500 IU/L), high eastern cooperative oncology group (ECOG) score (3-4), high neutrophil: lymphocyte ratio ( $>9)$, cancer type (lung), low pleural fluid $\mathrm{pH}(<7.28)$, and high sVEGFR-1 pleural fluid level [9-13].

However, none of the above stated predictors are sufficiently accurate, leaving patients exposed to errors in decision making. Adding to this insufficiency, conversely, in selected cancer type such as lung cancer, epidermal growth factor receptor (EGFR) mutation and EGFR-TKI therapy predicts longer survival $[14,15]$. Consequently, in search for a better predictor, LENT score was developed and recently published by Clive et al. [9] to risk-stratify unselected patients with MPE. LENT prognostic score is a composite score formed by the combination of pleural fluid LDH, ECOG status, serum NLR, and tumor type [9]. It classifies patients into low, moderate, and high-risk groups based on the duration of survival [9]. Ninety seven percent of patients belonging to high risk group reportedly die within 6 months when stratified based on the LENT scoring system [9].

Since LENT scoring system was developed for a diverse unselected population, we performed this study to assess the performance of LENT score in the "selected" population of lung adenocarcinoma. This is a common cancer which presents not uncommonly with MPE. We hypothesized that since survival trends show improvement in lung cancer, especially adenocarcinoma, by virtue of the discovery of cancer-driving mutations and targeted therapy, LENT scoring system may underestimate prognosis in this group of patients.

LENT Score Underestimate Prognosis in

MPE from Lung Adenocarcinoma

\section{Material and Methods}

The aim of this study was to assess the performance of LENT score in predicting prognosis in selected population of MPE secondary to lung adenocarcinoma.

Patients hospitalized and managed for MPE in the year 2012 were studied. Patients diagnosed with MPE from adenocarcinoma lung between January 1, 2012 and December 31, 2012 at our center were identified from our department database using the ICD code for MPE. Out of these, patients having MPE from adenocarcinoma lung were filtered out, followed by the review of electronic medical records. Data were collected on age, gender, side of pleural effusion, serum LDH, serum protein, pleural LDH, pleural protein, pleural fluid differential cell count, cytology, ECOG status, pleural biopsy result, and survival. All patients reviewed were managed as in-patients as only those presenting first time with the pleural effusion were assessed, and these required admission due to either symptoms or need for work-up such as thoracentesis and pleural drainage. Survival was calculated from the date of diagnosis of MPE and date of death. Follow-up data were available for all patients. LENT score was calculated for each patient according to the published literature [9]. Data were also analyzed by modifying the LENT score by substituting " 0 " for " 2 " in the "T" domain of the LENT score. Institutional Review Board approval was obtained for this study with the waiver of consent.

\section{Data Analysis}

We used SPSS software, version 17 (SPSS, Chicago, IL, USA) for all statistical analyses. The results were compared using a Wilcoxon 2 -sample test or Fisher exact test. $p$ values were 2 -sided and considered indicative of a significant difference if $<0.05$.

\section{Results}

A total of 100 patients with MPE were identified, of which MPE was secondary to lung adenocarcinoma in 70 patients. These were analyzed for the present study. There were no missing data for any of the variables as all patients underwent pleural fluid analysis in a standardized manner, with results keyed into the electronic system making the retrieval easy. Mortality data were also available for each patient with the help of national electronic health record system. All patients were well enough to receive some form of cancer-specific therapy regardless of the performance status.

The median (range) age was $72(38-92)$ years. There were $37(53 \%)$ males, and 39 (55.7\%) patients exhibited activating mutation in EGFR and received TKI's. The median LENT score was $5(2-7)$, and the overall survival was $241(4-1,216)$ days. The duration of follow-up was 7.9 (0.13-40) months (Table 1).

In sub-group analysis, the median (range) LENT score was $4(2-7)$, and $5(2-7)$ in the sub-group treated with EGFR-TKIs, and sub-group treated with conventional 
Table 1. Demographic features, clinical features, and pleural fluid biochemistry of patients with MPE from adenocarcinoma lung $(n=70)$

\begin{tabular}{lc}
\hline Variables & $n=70$ \\
\hline Age, years & $72(38-92)$ \\
Gender, male & $37(53)$ \\
Epidermal growth factor receptor mutation & $39(55.7)$ \\
Survival, days & $241(4-1,216)$ \\
Treated with TKI's & $39(55.7)$ \\
Treated with conventional chemotherapy & $31(44.2)$ \\
ECOG status & $2(0-4)$ \\
Pleural fluid lactate dehydrogenase & $929(267-3,800)$ \\
Blood neutrophil lymphocyte ratio & $4.8(1-59)$ \\
LENT score & $5(2-7)$ \\
\hline
\end{tabular}

Data is presented as $n(\%)$, or median (range).

MPE, malignant pleural effusion; ECOG, Eastern Cooperative Oncology Group; TKI’s, tyrosine kinase inhibitors.

chemotherapy $(p=0.44)$, respectively. The median (range) survival was $437(16-1,216)$ days, and $125(4-$ $1,043)$ days in the sub-group $(n=39)$ treated with EGFRTKIs, and sub-group $(n=31)$ treated with conventional chemotherapy ( $p=0.003)$, respectively (Fig. 1).

Nearly half of the patients, $51.4 \%$ (36/70), belonged to the high-risk category based on LENT score, and the other half, $48.5 \%$ (34/70), belonged to moderate-risk category, with none in low-risk category. Patients in the high-risk category, which is a LENT score $\geq 5(n=36)$, in the whole group, had a median survival of 190.5 days, and more than half, $52.7 \%$ (19/36), survived $>6$ months. In those belonging to the moderate-risk category, which is a LENT score $2-4(n=34)$, the median survival was 346 days, and $70.5 \%(24 / 34)$ survived $>6$ months. In the sub-group treated with targeted therapy $(n=39)$, $19(48.7 \%)$ had a LENT score of $\geq 5$, with a median survival of 530 days, and $73.6 \%(14 / 19)$ survived $>6$ months (Table 2).

Thirty nine (55.7\%) patients had activating mutation in EGFR in our cohort. EGFR mutations have been reported in $72.5 \%$ of patients with MPE from adenocarcinoma lung in the Korean population [16]. A positive association between EGFR mutation and the presence of MPE, suggesting a role of EGFR mutations in the formation of MPE has also been reported from China [17]. Due to this, we hypothesize that since significant number of patients of adenocarcinoma lung presenting with MPE will have EGFR mutation enabling longer survival, the " 2 " in the " $\mathrm{T}$ " domain of LENT score substituted with "0" will improve its accuracy. Hence, we decided to substitute "0" for "2" in the "T" domain of the LENT score. After substituting " 0 " for " 2 " in the "T" domain of LENT score, $4.2 \%(3 / 70)$ belonged to high-risk category, $70 \%$ (49/70), belonged to moderate-risk category, and $25.7 \%$ (18/70) belonged to low-risk category. The median survival and survival rate at 6 months in high-, moderate-, and low-risk groups was 113 days and $33.3 \%(1 / 3), 208$ days and $53 \%(26 / 49)$, and 491 days and $83.3 \%(15 / 18)$, respectively (Table 2$)$. In the sub-group treated with targeted therapy $(n=39), 1$ had a LENT score of $\geq 5,27$ had a LENT score of $2-4$, and 11 had a LENT score of $0-1$ with a median survival and survival rate at 6 months of 113 days, and $0 \%, 357$ days and $77.7 \%$, and 655 days and $91 \%$, respectively.

\section{Discussion}

We illustrated that the actual survival in patients having MPE from lung adenocarcinoma was higher than predicted by the LENT score. Modification of the LENT score by substituting " 0 " for " 2 " in the " $\mathrm{T}$ " domain improved its accuracy in this cohort.

LENT score was developed as a risk stratification system to predict survival in MPE, calculated based on pleural fluid LDH, ECOG status, serum NLR, and tumor type [9]. Patients with a combination of high pleural fluid LDH, high ECOG status, high NLR, and cancer type other than mesothelioma, renal cell carcinoma, hematological cancer, gynecological cancer, and breast cancer, score highest according to the LENT scoring system [9]. Lung cancer was assigned highest risk category among cancer types based on the poor median survival of 74 (60-92) days in the validation cohort of the LENT scoring system [9].

However, with the discovery of mutational drivers of lung cancer such as EGFR mutation, and the advent of EGFR-TKI therapy since 2009, survival rates have improved dramatically in lung cancer patients carrying activating EGFR mutation [18-22]. Prior to the advent of TKI's, the median survival used to be $6.5-8$ months in patients with MPE from lung cancer [23]. However, the median survival in those receiving TKI's has been reported as 14 months with a survival rate at 1 year of $52.9 \%$ [15]. These findings are consistent with the findings in our cohort.

The median survival in our cohort was 241 days, which was better than the survival of 74 days reported by Clive et al. [9] in their cohort. Among those who received TKI's, the median survival was 437 days, even higher than the survival reported in the "lung cancer" group by Clive et al. [9] in their LENT validation cohort. The median survival in our whole cohort was close to mesothelioma, the 
Table 2. Comparison of median survival and survival rate at 6 months based on LENT score in the current study, with the published literature [9]

$\begin{array}{lll}\begin{array}{l}\text { Clive et al. [9] } \\ (n=203)\end{array} & \begin{array}{l}\text { Current study } \\ (n=70)\end{array} & \begin{array}{l}\text { Current study } \\ (n=70)\end{array} \\ \text { (LENT score) } & \text { (LENT score) } & \text { (modified LENT score) }\end{array}$

\begin{tabular}{|c|c|c|c|}
\hline \multicolumn{4}{|l|}{ Survival, days } \\
\hline High risk (LENT $\geq 5$ ) & $44(22-77)$ & $190.5(4-1,216)$ & $113(27-20$ \\
\hline Moderate risk (LENT 2-4) & $130(47-467)$ & $346(18-1,193)$ & $208(4-1,21$ \\
\hline Low risk (LENT 0-1) & $319(228-549)$ & - & $491(32-1,1$ \\
\hline \multicolumn{4}{|l|}{ Survival rate at 6 months } \\
\hline High risk (LENT $\geq 5$ ) & $3^{*}$ & $19(52.7)$ & $1(33.3)$ \\
\hline Moderate risk (LENT 2-4) & $47^{*}$ & $24(70.5)$ & $26(53)$ \\
\hline Low risk (LENT 0-1) & $86^{*}$ & - & $15(83.3)$ \\
\hline
\end{tabular}

Data presented in median (range), and percentages or $n(\%)$.

* Number of patients not provided in the LENT paper.

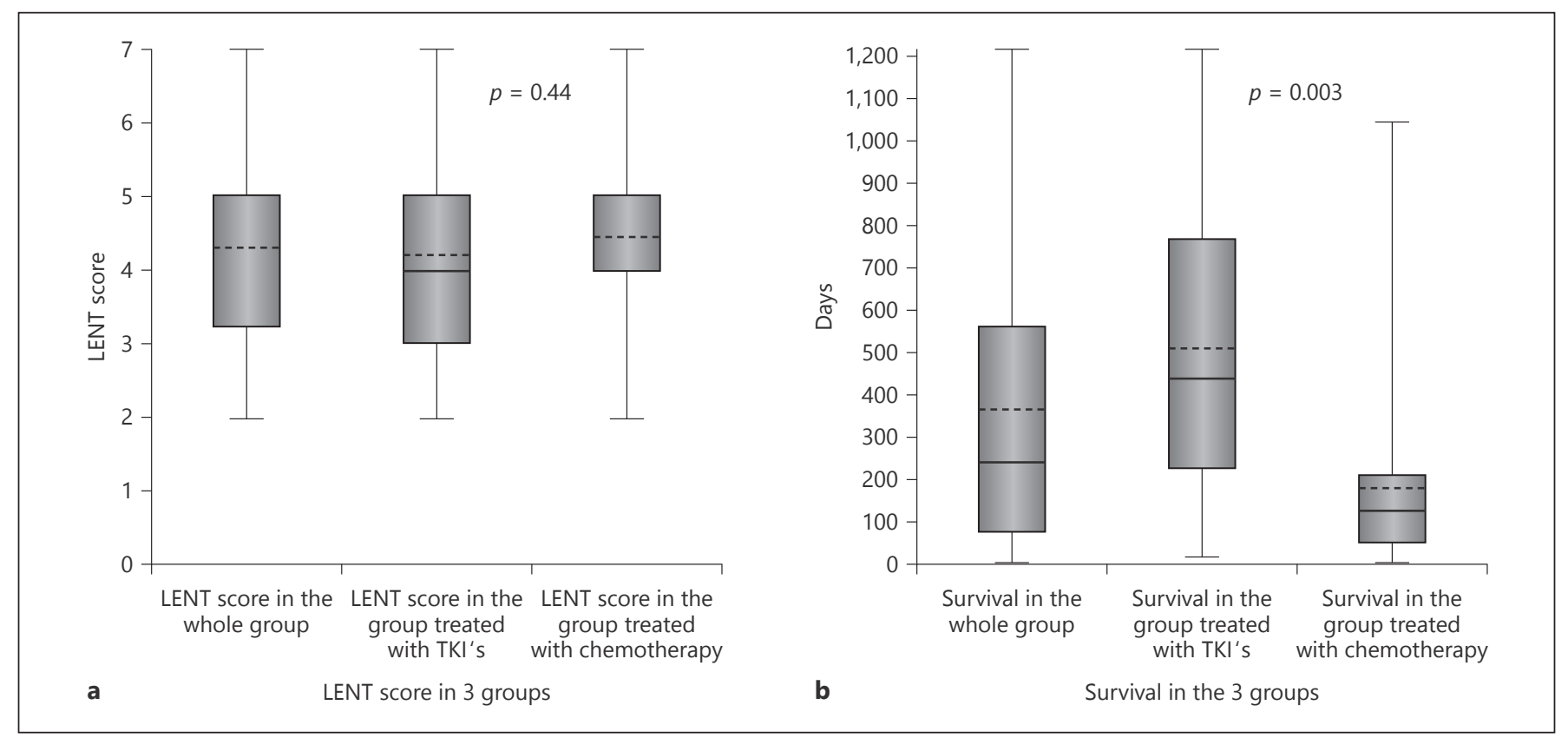

Fig. 1. a Box plot showing the median LENT score in the whole group, sub-group treated with EGFR-TKIs, and sub-group treated with conventional chemotherapy. b Box plot showing the median (solid horizontal line) survival in the whole group, sub-group treated with targeted therapy (EGFR-TKIs), and sub-group treated

cancer type associated with best survival in the study by Clive et al. [9]. Furthermore, the survival in the sub-group of patients who received TKI's in our cohort was higher than even mesothelioma.

Based on LENT score, most patients in our cohort fell in the high-risk or moderate-risk category with none in with conventional chemotherapy. Despite similar LENT scores between whole group, sub-group treated with EGFR-TKIs, and subgroup treated with conventional chemotherapy, survival was significantly longer in the TKI group compared to chemotherapy group. TKI, tyrosine kinase inhibitor. the low-risk group. Based on this score, the survival rate at 6 months of patients falling in these categories should be 3,47 , and $86 \%$, respectively [9]. However, the proportion of patients surviving $>6$ months in the high-risk and moderate-risk groups of our cohort was 52.7 and $70.5 \%$, respectively, much higher than that reported of 3 
and $47 \%$. In patients who belonged to the high-risk group and were treated with TKI's, the survival rate was even higher, $73.6 \%$ at 6 months, much higher than the 3\% quoted in the LENT study.

This study indicates that it may be more appropriate for patients with lung adenocarcinoma and activating EGFR mutation to be assigned the score of "0" instead of " 2 " in the "T" domain of the LENT score. Without this, the LENT score may underestimate prognosis and falsely assign poor prognosis to this group of patients depriving them of appropriate treatment modalities.

When we re-calculated the LENT score by assigning " 0 " instead of " 2 " in the T domain of the LENT scoring system, the risk profile changed. The least number of patients now belonged to the high-risk category as compared to when the score of " 2 " was assigned to the " $T$ " domain. The highest number of patients (two-thirds) now belonged to moderate-risk category, and a quarter belonged to the low-risk category. The proportion of patients surviving $>6$ months in the high-risk, moderaterisk, and low-risk groups of our cohort was 33.3, 53, and $83.3 \%$ with the modification of the LENT scoring system.

Hence, the LENT score was found to have limitations in predicting survival in patients having MPE from lung adenocarcinoma, especially in those treated with EGFR TKI's. On the same note, while it can be argued that assigning " 0 " instead of " 2 " in the " $\mathrm{T}$ " domain may be more appropriate for patients with adenocarcinoma and EGFR mutation, for those with adenocarcinoma without EGFR mutation, assigning " 1 " instead of " 0 " in place of " 2 " may be more appropriate, and is a possible area to explore in the future.

Lung cancer being the cancer associated with the worst prognosis, has attracted significant progress in the development of novel therapies in recent years as a battle against it. Identification of several mutational drivers such as EGFR, ALK, ROS1 etc., with corresponding targeted therapies have significantly changed the survival statistics of lung cancer. In addition, immunotherapy carries potential to further improve the survival in lung cancer patients [24]. In view of these advances, the LENT score may indeed need modification for this group of patients. Similar findings have been published by investigators from Japan who reported $14(87.5 \%)$ patients in the high-risk group based on LENT score but could use TKI's for survival longer than 6 months [25].

However, it is noteworthy to highlight that the prevalence of EGFR mutation in Singapore is $40 \%$, whereas the prevalence in countries LENT was derived from is $12 \%$ in UK, $12 \%$ in Australia, and 15\% in Netherland [26]. This imposes geographical limitations on applying the LENT score. Authors would like to draw the readers' attention to this fact when interpreting our findings. Nevertheless, our study elucidates the limitations of the prognostic scores, demonstrating that they need to be revised and revalidated with the therapeutic advances to keep up with them. Future studies with prospective validation in other Asian countries, and impact analysis to see if the LENT score really alters outcomes will help to improve the clinical decision making [27].

Our study has the limitation of being a single center experience with a retrospective observational design. However, the main strength of our study is that it was conducted on a homogenous population of adenocarcinoma lung, thus increasing the precision with which it can be applied to this group of patients. Additionally, patients with lung adenocarcinoma with MPE have a higher EGFR mutation detection rate compared to patients with lung adenocarcinoma presenting as a solitary mass or nodule. The EGFR mutation rate in MPEs of lung adenocarcinoma has been reported to be as high as $70 \%$ [28]. Consequently, greater proportions of patients with adenocarcinoma and a MPE phenotype are eligible for TKI therapy. Hence, based on our findings, modifying or not modifying the LENT score may impact $70 \%$ of patients with MPEs from lung adenocarcinoma.

In conclusion, the actual survival in patients having MPE from lung adenocarcinoma is higher than that predicted by LENT score. The LENT score may underestimate prognosis in such patients and needs to be modified to improve its accuracy in this group of patients.

\section{Acknowledgements}

Authors would like to thank Ms. Ivy Yu Ling Ling for her valuable contribution in the administrative work and preparing the figures.

\section{Ethics Approval and Consent to Participate}

Ethics approval was obtained from the institutional review board.

\section{Consent for Publication}

Not applicable. 


\section{Availability of Data and Materials}

The datasets used and/or analyzed during the current study are available from the corresponding author on reasonable request.

\section{Funding Source}

No funding was used for this study.

\section{Disclosure Statement}

J.A., A.V., R.S.D., and R.W.L. have no potential conflicts of interest to report.

\section{Authors' Contributions}

A.V. and J.A. conceived the study, collected the data, analyzed the data, and drafted the manuscript. R.S.D. collected and analyzed the data, and R.W.L. reviewed and revised the study.

\section{References}

1 Heffner JE, Klein JS: Recent advances in the diagnosis and management of malignant pleural effusions. Mayo Clin Proc 2008;83: 235-250.

2 Shaw PH, Agarwal R: WITHDRAWN: Pleurodesis for malignant pleural effusions. Cochrane Database Syst Rev 2013; 11:CD002916.

3 Wozniak AJ, Gadgeel SM: Clinical presentation of non-small cell carcinoma of the lung; in Pass HI, Carbone DP, Johnson DH, Minna JD, Scagliotti GV, Turrisi AT (eds): Principles and Practice of Lung Cancer, ed 4. Philadelphia, Lippincott Williams \& Wilkins, 2010,pp 327-340.

4 Dresler CM, Olak J, Herndon JE 2nd, et al: Cooperative Groups Cancer and Leukemia Group B; Eastern Cooperative Oncology Group; North Central Cooperative Oncology Group; Radiation Therapy Oncology Group: Phase III intergroup study of talc poudrage vs talc slurry sclerosis for malignant pleural effusion. Chest 2005;127:909-915.

5 Shaw P, Agarwal R: Pleurodesis for malignant pleural effusions. Cochrane Database Syst Rev 2004;1:CD002916.

6 Reddy C, Ernst A, Lamb C, Feller-Kopman D: Rapid pleurodesis for malignant pleural effusions: a pilot study. Chest 2011;139:14191423.

7 Rintoul RC, Ritchie AJ, Edwards JG, et al: Efficacy and cost of video-assisted thoracoscopic partial pleurectomy versus talc pleurodesis in patients with malignant pleural mesothelioma (MesoVATS): an open-label, randomised, controlled trial. Lancet 2014;384: 1118-1127.

8 Gupta D, Ross K, Piacentino V 3rd, et al: Use of LeVeen pleuroperitoneal shunt for refractory high-volume chylothorax. Ann Thorac Surg 2004;78:e9-e12.

9 Clive AO, et al: Predicting survival in malignant pleural effusion: development and validation of the LENT prognostic score. Thorax 2014;69:1098-1104.
10 Zamboni MM, da Silva CT Jr, Baretta R, Cunha ET, Cardoso GP: Important prognostic factors for survival in patients with malignant pleural effusion. BMC Pulm Med 2015; 15:29.

11 Anevlavis S, Kouliatsis G, Sotiriou I, et al: Prognostic factors in patients presenting with pleural effusion revealing malignancy. Respiration 2014;87:311-316.

12 Heffner JE, Nietert PJ, Barbieri C: Pleural fluid $\mathrm{pH}$ as a predictor of survival for patients with malignant pleural effusions. Chest 2000; 117:79-86

13 Hooper CE, Elvers KT, Welsh GI, et al: VEGF and sVEGFR-1 in malignant pleural effusions: association with survival and pleurodesis outcomes. Lung Cancer 2012;77:443-449.

14 Kasapoglu US, Arınç S, Gungor S, et al: Prognostic factors affecting survival in non-small cell lung carcinoma patients with malignant pleural effusions. Clin Respir J 2015;10:791799.

15 Wu SG, Yu CJ, Tsai MF, et al: Survival of lung adenocarcinoma patients with malignant pleural effusion. Eur Respir J 2013;41:14091418.

16 Yang J, Lee OJ, Son SM, et al: EGFR mutation status in lung adenocarcinoma-associated malignant pleural effusion and efficacy of EGFR tyrosine kinase inhibitors. Cancer Res Treat 2017, Epub ahead of print.

17 Zou J, Bella AE, Chen Z, et al: Frequency of EGFR mutations in lung adenocarcinoma with malignant pleural effusion: implication of cancer biological behaviour regulated by EGFR mutation. J Int Med Res 2014;42:11101117.

18 Mok TS, Wu YL, Thongprasert S, et al: Gefitinib or carboplatin-paclitaxel in pulmonary adenocarcinoma. N Engl J Med 2009;361: 947-957.

19 Brugger W, Triller N, Blasinska-Morawiec M, et al: Prospective molecular marker analyses of EGFR and KRAS from a randomized, placebo-controlled study of erlotinib mainte- nance therapy in advanced non-small-cell lung cancer. J Clin Oncol 2011;29:4113-4120.

20 Zhu CQ, da Cunha Santos G, Ding K, et al: Role of KRAS and EGFR as biomarkers of response to erlotinib in National Cancer Institute of Canada Clinical Trials Group Study BR.21. J Clin Oncol 2008;26:4268-4275.

21 Douillard JY, Shepherd FA, Hirsh V, et al: Molecular predictors of outcome with gefitinib and docetaxel in previously treated nonsmall-cell lung cancer: data from the randomized phase III INTEREST trial. J Clin Oncol 2010;28:744-752.

22 Shigematsu H, Lin L, Takahashi T, Nomura $\mathrm{M}$, et al: Clinical and biological features associated with epidermal growth factor receptor gene mutations in lung cancers. J Natl Cancer Inst 2005;97:339-346.

23 Sugiura S, Ando Y, Minami H, Ando M, Sakai S, Shimokata K: Prognostic value of pleural effusion in patients with non-small cell lung cancer. Clin Cancer Res 1997;3:47-50.

24 Rijavec E, Genova C, Alama A, et al: Role of immunotherapy in the treatment of advanced non-small-cell lung cancer. Future Oncology 2014;10:79-90.

25 Takahashi T, Naito D, Wakatsuki Y, et al: Validation of the LENT score in Japanese sample: the impact of EGFR-TKI. ERJ 2015; 46:PA4328.

26 Midha A, Dearden S, McCormack R: EGFR mutation incidence in non-small-cell lung cancer of adenocarcinoma histology: a systematic review and global map by ethnicity (mutMapII). Am J Cancer Res 2015;5:28922911.

27 Barnes SA: Impact Analysis. Warwick Institute for Employment Research. https://warwick.ac.uk/fac/soc/ier/ngrf/effectiveguidance/impact/assessing/outcomes/paper/ (accessed on February 17, 2018).

28 Wu SG, Gow CH, Yu CJ, et al: Frequent epidermal growth factor receptor gene mutations in malignant pleural effusion of lung adenocarcinoma. Eur Respir J 2008;32:924-930. 